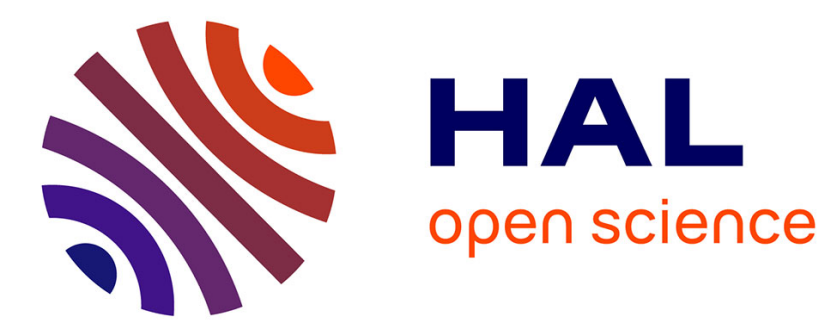

\title{
Sharp waveguide bends induced by spatial solitons
}

R. Jager, S.-P. Gorza, Cyril Cambournac, M. Haelterman, M. Chauvet

\section{To cite this version:}

R. Jager, S.-P. Gorza, Cyril Cambournac, M. Haelterman, M. Chauvet. Sharp waveguide bends induced by spatial solitons. Applied Physics Letters, 2006, 88, 3 p. 10.1063/1.2172649 . hal-00169242

\section{HAL Id: hal-00169242 \\ https://hal.science/hal-00169242}

Submitted on 17 Sep 2007

HAL is a multi-disciplinary open access archive for the deposit and dissemination of scientific research documents, whether they are published or not. The documents may come from teaching and research institutions in France or abroad, or from public or private research centers.
L'archive ouverte pluridisciplinaire HAL, est destinée au dépôt et à la diffusion de documents scientifiques de niveau recherche, publiés ou non, émanant des établissements d'enseignement et de recherche français ou étrangers, des laboratoires publics ou privés. 


\title{
Sharp waveguide bends induced by spatial solitons
}

Robert Jäger, Simon-Pierre Gorza, Cyril Cambournac, Marc Haelterman

Université libre de Bruxelles, Service d'optique et acoustique, B-1050 Bruxelles.*

\author{
Mathieu Chauvet \\ Institut Femto-ST, Département d'optique P-M. Duffieux, \\ UMR CNRS/Université de Franche-Comté 6174, F-25030 Besançon cedex. ${ }^{\dagger}$
}

(Dated: January 16, 2006)

We experimentally demonstrate the ability of a self-guided laser beam to induce waveguides with sharp bends. The beam is a two-dimensional photorefractive screening-photovoltaic bright spatial soliton generated inside a biased lithium-niobate crystal shaped as a prism. The soliton robustness against total internal reflections is shown to leave place to a low-loss unimodal waveguide undergoing multiple zero-radius $90^{\circ}$ turns.

*URL: http://www.ulb.ac.be/polytech/soa

†URL: http://lopmd.univ-fcomte.fr/onl 
The study of spatial optical solitons has become a major area in the field of nonlinear optics, as witness the numerous textbooks and the steady emulation of the scientific community in this field of research (see, e.g., Refs. [1-4]). Such an activity is firstly motivated by the fundamental aspects of the study on optical solitons and related instabilities for a better understanding of nonlinear dynamical systems, ubiquitous in nature. Besides, research on spatial optical solitons is also deeply stimulated by the promising benefits that could bring their use as the building blocks of future all-optical signal-processing devices [5,6]. This awaited breakthrough in engineering sciences stems from the concept of self-induced waveguide that is at the heart of the phenomenon of soliton-like beam propagation in optics. This explains why a significant experimental effort is aimed at demonstrating the practical feasibility of all-optical devices based on spatial solitons.

Owing to its numerous physical properties (e.g., electrooptic, ferroelectric, pyroelectric, or piezoelectric) and its availability in good optical quality, lithium niobate $\left(\mathrm{LiNbO}_{3}\right)$ is largely employed in optoelectronics. Moreover, at visible wavelengths $\mathrm{LiNbO}_{3}$ exhibits a strong photorefractive (PR) effect which is already used for applications such as volume holographic data storage [7] or the realization of Bragg filters [8]. Currently, part of the research activity on $\mathrm{LiNbO}_{3}$ is aimed at optically inscribing waveguides which constitutes an appealing alternative to existing complex technological process. For this purpose, techniques such as, e.g., UV-beam exposure [9] or the propagation of either 1-D or 2-D PR soliton-like beams can be used. The latter technique offers a very simple means for the inscription of unimodal waveguides without any deterioration in the crystalline quality of the host crystal [10]. Up to very recently, however, only dark or vortex spatial photovoltaic solitons were observed in $\mathrm{LiNbO}_{3}$ [11, 12]. Nevertheless, as regards waveguide-inscription purposes, bright soliton-like beams provide easier means of imprinting 2-D waveguides. Fazio et al. recently demonstrated that $\mathrm{LiNbO}_{3}$ is also able to support bright 2-D soliton-like beams when electrically biased [13], thus demonstrating generation of 2-D bright spatial solitons of screening-photovoltaic kind [14]. Realization of complex waveguides trajectory with zero-radius turns is a challenging goal which is crucial, particularly in the context of optical interconnects and high-density integrated optics. In this Letter we propose to use reflection of spatial solitons to create such complex waveguides in $\mathrm{LiNbO}_{3}$. To our best knowledge only a limited number of experimental works highlights the internal reflection of a spatial soliton at the interface between a nonlinear medium and a linear one $[16,17]$. In this work we first demonstrate that photovoltaic-screening photorefractive spatial solitons are robust against total internal reflection (TIR). Soliton-like beams are shown to be able to undergo stable $90^{\circ}$ abrupt turns inside a prismatic undoped photonic-grade $\mathrm{LiNbO}_{3}$ crystal. We then demonstrate that the soliton leave place to an efficient waveguide with zero-radius bends. Evaluation of the guiding properties of the photo-induced waveguide reveals that the photo-induced waveguide supports transmission of higher-wavelength light with very low loss. We believe that such a technique to inscribe can be useful, for instance, for the development and integration of 
bulk all-optical interconnects and for high-density integrated optics.

The experimental set-up is depicted in Figure 1.a. To show that spatial soliton in $\mathrm{LiNbO}_{3}$ is able to withstand TIR and, subsequently, to give rise to waveguides with zero-radius bends we use a $\mathrm{LiNbO}_{3}$ right-angle isosceles prism as sketched in Figure 1.b. This prism which has a $10 \mathrm{~mm}$ base edge and $0.5 \mathrm{~mm}$ thickness is cut from a $0.5 \mathrm{~mm}$-thick photonic-grade $z$-cut $\mathrm{LiNbO}_{3}$ wafer. Electrodes are deposited on $z$-faces whereas other faces are optically polished. A continuous-wave $532 \mathrm{~nm}$ beam is delivered from a frequency-doubled Nd:YAG laser. The beam is split into two parts, with $90 \%$ reflected light being used as a background illumination $\left(I_{\mathrm{b}}\right)$ to artificially tune the dark intensity of the crystal. The remaining transmitted light is used to form a soliton beam, with a linear polarization parallel to the $c$-axis (optical $z$-axis). Both beams are made mutually incoherent by use of orthogonal linear-polarization states. Given that a significant static electric potential difference is applied to the prism, it is bathed into a silicon-oil-filled tank in order to avoid electrical arcing in the surrounding medium. $\mathrm{LiNbO}_{3}$ has an extraordinary refractive index of 2.2 whereas the oil's one is about 1.4. These values of refractive indices give a critical incident angle for TIR of about $39.5^{\circ}$, which a fortiori allows for TIR at $45^{\circ}$, that is, for our experimental conditions of a light incidence normal to the prism's hypotenuse. Translation and rotation stages allow tuning of crystal position in all directions and its orientation to achieve normal incidence of incoming light. Similarly than in reference [13], conditions for soliton formation are the followings: The static bias electric field is $35 \mathrm{kV} / \mathrm{cm}$, the background intensity is $I_{\mathrm{b}} \approx 0.4 \mathrm{~mW} / \mathrm{cm}^{2}$, and the soliton power is $P_{\mathrm{s}} \approx 20 \mu \mathrm{W}$, so that, for our experimental beam widths, the corresponding peak intensity is 1500 times more intense than the background intensity. The high $I_{\mathrm{s}} / I_{\mathrm{b}}$ ratio implies that the soliton beams form in quasi-steady-state regime. In this situation the soliton width is expected to be independent of the background intensity. In our experiment presence of the background irradiance is to prevent beam bending as it was observed in reference [18]. Figure 2 shows a typical observation of soliton formation and robustness to TIRs. The profiles are analyzed using a CCD camera in combination with imaging optics that allow the observation of the prism's base (i.e. the input and output beams at will) by simply translating an objective (see Fig. 1.a). The input beam is focused on the base of the prism to a $15 \mu \mathrm{m}$ FWHM waist (Fig. 2.a). It then propagates perpendicular to the input face and experiences two TIRs at right angle before exiting the sample (Figs. 2.b and c). Note that although an illumination grating is present at each reflection site due to the interference between the incoming and reflected beams the associated photorefractive index grating can be neglected because of the an inadequate electro-optic configuration along with a small overlap region. In the linear regime, we first observe that the beam strongly diffracts during the five-diffraction-length-long $(1 \mathrm{~cm})$ trip inside the crystal but, in addition, beam distortion attributed to poorly polished faces is also visible (Fig. 2.b). Even so, once the bias electric field is applied the light gradually focuses to finally give rise to a solitonic beam (Fig. 2.c). The soliton-beam formation time is about 20 minutes which can be 
lowered, for example, by increasing the intensity or the applied bias [13]. Nevertheless, this result demonstrates that photorefractive spatial solitons in $\mathrm{LiNbO}_{3}$ are robust against multiple internal reflections. We then check that the soliton-induced waveguide is able to guide light at a different wavelength. Figure 3 shows the linear guidance of a linearly polarized (along $c$-axis) $633 \mathrm{~nm}$ beam coming from a cw He:Ne laser in the complex waveguide previously inscribed inside the prism. Note that both the $532 \mathrm{~nm}$ soliton beam and the applied potential difference are switched off during the guiding test. It is clearly seen that the inscribed tubular waveguide allows efficient light transmission even in the two TIRs area. Thanks to the high value of the dielectric response time of $\mathrm{LiNbO}_{3}$ inscribed waveguides can last up to months when illuminated with low intensity and/or long wavelength [13]. We observe that the guided mode at $633 \mathrm{~nm}$ (Fig. 3.d) is wider than for shorter wavelength (Fig. 3.b), as expected from theory. Thanks to the high purity of the undoped $\mathrm{LiNbO}_{3}$ crystal, propagation loss in the waveguide is very low. We measured an overall light transmission in the waveguide of about $96 \%$ taking into account Fresnel reflections and loss in silicon oil which gives an absorption coefficient of about $0.04 \mathrm{~cm}^{-1}(\simeq 0.17 \mathrm{~dB} / \mathrm{cm})$. Moreover, the low light noise present in the vicinity of the waveguide at the exit face (see contour plot of Fig. 3.d) reveals that a very good coupling efficiency is achieved into the waveguide. Notwithstanding, we attribute most of this noise to scattering at the two TIR surfaces (some scattered light is indeed visible in the right-top side of the beam in Figure 2.c). Careful polishing should consequently reduce these imperfections and further improve light transmission in the waveguide.

In conclusion, we showed experimentally that a 2-D bright soliton-like beam is able to form a long-lived unimodal waveguide with two zero-radius $90^{\circ}$ turns using TIRs inside a $\mathrm{LiNbO}_{3}$ crystal. Moreover, the light induced waveguide possesses excellent guiding properties. This result establishes that photorefractive solitons with complex trajectories offer an unique way to fabricate efficient waveguides with sharp direction changes, like zero-radius bends. Besides versatility and reconfigurability, self-guided beams do not necessitate costly equipment contrary to standard fabrication techniques commonly used for integrated-optics applications. Moreover, the tubular waveguides created are expected to be compatible with optical fiber without use of micro-lens. Finally we believe that the present study paves the way for the fabrication of volume soliton-based complex guiding integrated structures not only in lithium-niobate crystals but also more generally in PR host crystals.

The authors wish to acknowledge the support of the Interuniversity Attraction Poles program IAP V-18 PHOTONnetwork of the Belgian Science Policy (cf. http://www.tona.vub.ac.be/photon/). This work has also been supported by the French Ministry of Education and Research through an Action Concertée Nanosciences 2004 (project NR137). 
[1] G. I. Stegeman and M. Segev, Science 286, 1518 (1999).

[2] A. D. Boardman and A. P. Sukhorukov (Eds.), Soliton-Driven Photonics, NATO Sciences Series II vol.31 (Kluwer Academic Publishers, Dordrecht, 2001).

[3] S. Trillo and W. E. Torruellas (Eds.), Spatial Solitons (Springer, Berlin, 2001).

[4] Yu. S. Kivshar and G. P. Agrawal, Optical Solitons (Academic Press, San Diego, 2003).

[5] A. W. Snyder and F. Ladouceur, Opt. Photon. News 10, 35 (1999).

[6] Yu. S. Kivshar and G. I. Stegeman, Opt. Photon. News 13, 59 (2002).

[7] G. W. Burr, C. M. Jefferson, H. Coufal, M. Jurich, J. A. Hoffnagle, R. M. Macfarlane, and R. M. Shelby, Opt. Lett. 26, $444(2001)$.

[8] J. Hukriede, D. Kip, and E. Krätzig, Appl. Phys. B 72, 749 (2001).

[9] S. Mailis, C. Riziotis, I. T. Wellington, P. G. R. Smith, C. B. E. Gawith, and R. W. Eason, Opt. Lett. 28, 1433 (2003).

[10] M.-F. Shih, Z. Chen, M. Mitchell, M. Segev, H. Lee, R. S. Feigelson, and J. P. Wilde, J. Opt. Soc. Am. B 14, 3091 (1997).

[11] M. Taya, M. C. Bashaw, M. M. Fejer, M. Segev, and G. C. Valley, Phys. Rev. A 52, 3095 (1995).

[12] Z. Chen, M. Segev, D. W. Wilson, R. E. Muller, and P. D. Maker, Phys. Rev. Lett. 78, 2948 (1997).

[13] E. Fazio, F. Renzi, R. Rinaldi, M. Bertolotti, M. Chauvet, W. Ramadan, A Petris, and V. I. Vlad, Appl. Phys. Lett. 85, 2193 (2004).

[14] L. Jinsong and L. Keqing, J. Opt. Soc. Am. B 16, 550 (1999).

[15] D. N. Christodoulides and T. H. Coskun, Opt. Lett. 21, 1220 (1996).

[16] E. Alvarado-Méndez,R. Rojas-Laguna, J.G. Avina-Cervantes, M. Torrres-Cisneros, J.A. Andrade-Lucio, J.C. PedrazaOrtega,E. A. Kuzin, J.J. Sánchez-Mondragón,V. Vysloukh, Opt. Commun. 193, 267 (2001).

[17] F. Baronio, C. De Angelis, P-H. Pioger, V. Couderc, and A. Barthélémy, Opt. Lett. 29, 986 (2004).

[18] M. Chauvet, V. Coda, H. Maillotte, E. Fazio, and G. J. Salamo, Opt. Lett. 30, 1977 (2005). 


\section{List of Figures}

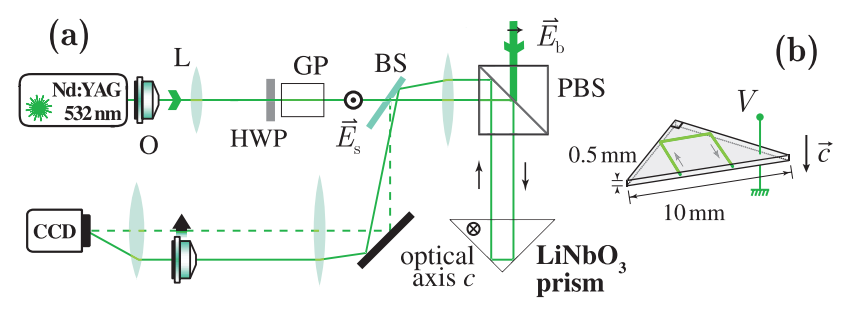

FIG. 1:Experimental arrangement. Thick line : background illumination, thin line : soliton beam, dashed line : soliton beam reflected at the prism input. - (a) Set-up (top view): O: microscope objectives, L: lenses, BS: beam-splitters, HWP: half-wave plates, GP: Glan polarizer, PBS: polarizing beam-splitter; (b) Sketch of the right-angle $\mathrm{LiNbO}_{3}$ prism.

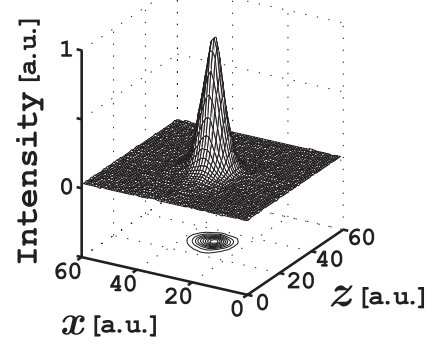

(a) INPUT BEAM

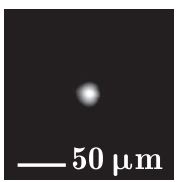

(b) OUTPUT BEAM
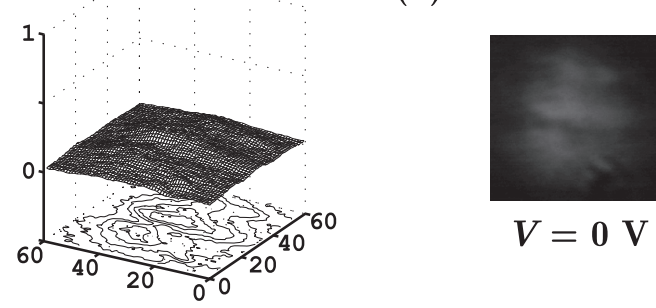

$V=0 \mathrm{~V}$

(c) OUTPUT BEAM
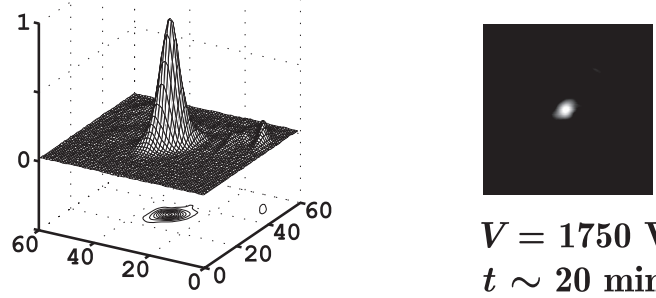

$V=1750 \mathrm{~V}$

$t \sim 20 \mathrm{~min}$

FIG. 2:Observation of the robustness of a soliton-like beam to two total internal reflections (experimental images and corresponding 3-D meshes and 2-D isoline projections) - (a) Input 15 pm FWHM beam; (b) Output beam in linear propagation regime (no applied field) after two total internal reflections; (c) Output beam in soliton regime (applied field). 


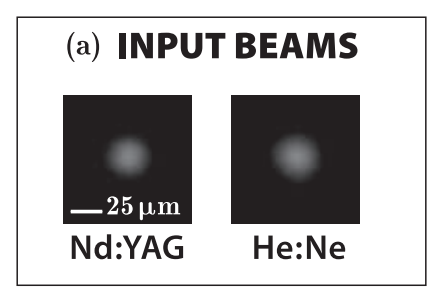

\section{OUTPUT BEAMS}
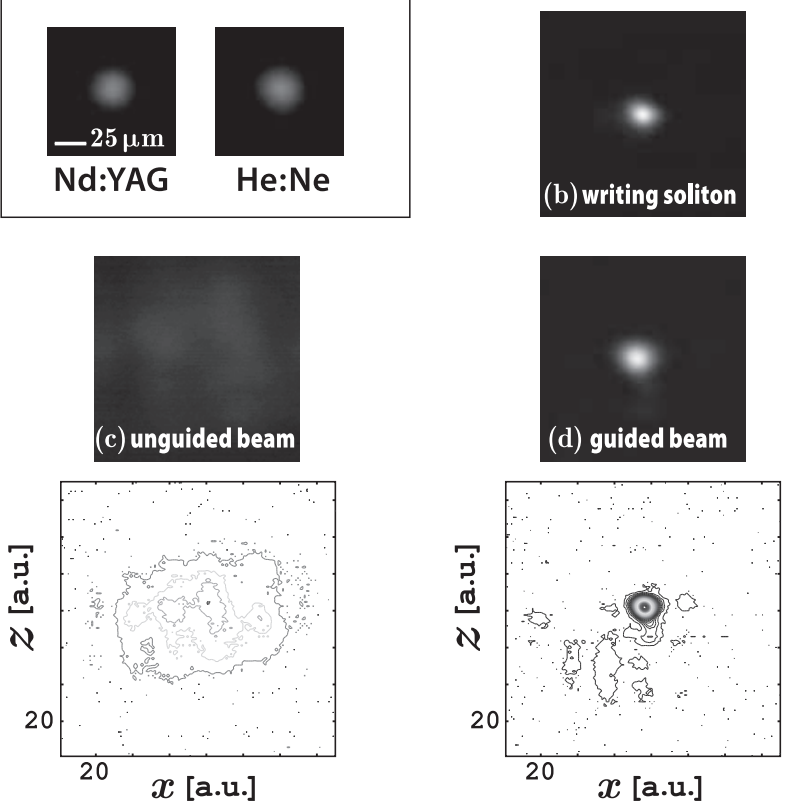

FIG. 3:Guided propagation at $633 \mathrm{~nm}$ in a soliton inscribed waveguide with two $90^{\circ}$ turns. — (a) soliton beam (Nd:YAG) and probe beam(He:Ne) at the input; (b) Soliton beam at the output (applied field); (c) image and contour plot of the probe beam at the output when injected away from the inscribed waveguide and (d) when probe beam is injected in the soliton induced waveguide (no applied field). 